\title{
Research Progress in the Relationship between Infant Temperament and Sleep
}

\author{
Jing JI, Hong-Ling ZHANG*, Ling QI \\ College of Health Science and Nursing \\ Wuhan Polytechnic University \\ WHPU \\ Wuhan, China
}

\author{
Ao-Shuang XIAO, Rong NIE, Qi LI, Yu MING \\ College of Health Science and Nursing \\ Wuhan Polytechnic University \\ WHPU \\ Wuhan, China
}

\begin{abstract}
Sleep plays an important role in infant growth and development. In this paper, the literature on the relationship between temperament and sleep was summarized and analyzed at home and abroad to understand the differences in sleep performance of infants with different temperament types, and to summarize the influence of temperament on sleep. To provide a reference for the clinical study of related problems in the future.
\end{abstract} sleep

Keywords-infant; temperament; behavioral development;

Temperament is the external manifestation of infants' behavioral development. Some studies have shown that temperament is closely related to the development of infants' psychological behavior, and temperament is also related to infants' sleep behavior, moreover, infants with different temperament types have different sleep behaviors [1]. In China, there are many studies on sleeping of school-age children, but only a few studies on sleep in infants. Also, there are few reports on the correlation between infant temperament characteristics and sleep. Studies at home and abroad have found that the relationship between infant sleep behavior and temperament showed that infants' sleeping behavior is closely related to temperament type, and infants with different temperament types have different sleep behaviors [2]. Studies abroad have found that in infants times, difficult temperament is related to the short duration of sleep of infant, and it also is related to sleep infant problems. Children with difficult temperament types tend to be more sensitive, irritable and vulnerable by changes in the external environment, with disturbances and intense reactions, and such infants are more likely to wake up at night [3]. Studies have pointed that infants with poor sleep quality have a higher frequency of difficult temperament than infants with good sleep quality, and are more likely to lose their temper and get higher score in the test of emotional and behavioral problems [4]. This paper mainly reviewed the temperament characteristics, temperament type, sleep characteristics and sleep time of infants.

\section{INFANT TEMPERAMENT TYPES AND CHARACTERISTICS}

Temperament is a relatively obvious and stable personality characteristic that is the earliest manifestation of infants after birth. It is the external manifestation of behavioral development of infants and children. Temperament-related research began in
Thomas and Chess in the United States, and they proposed a theory, namely nine dimensions of temperament: activity level, rhythm, avoidance, adaptability, response intensity, emotional essence, persistence, attention dispersion, and response threshold. The NYLS team also divided infant temperament into five types: easy type, middle near easy type, middle near difficulty type, slowly start type and difficult type. Most of the current studies use these two definitions to evaluate temperament. On the basis of a large number of investigations and studies, domestic scholars have divided the temperament types of infants into three types, that is easy, difficult, and retarded. Among them, $40 \%$ of infants belong to easy-type, difficult-type infants account for about $10 \%$, and retarded type account for about $15 \%$, and the remain $35 \%$ of infants often have several types of mixed characteristics [5]. Temperament involves the whole brain's neural activity, mainly the cerebral cortex in the first half and the limbic system. The basis of temperament production mechanism is the structural and functional characteristics of neural tissue and the diverse combinations of them [6].

\section{CHARACTERISTICS AND SIGNIFICANCE OF INFANT SLEEP}

Sleep is an important physiological process of human beings, and sleep is the basic activity of the brain in early development of infants. Therefore, infants need to longer sleeping time. The brain has a remodeling feature in the early stages of development, and by remodeling, the brain function can be improved. However, if missing in the early stage of brain development, remodeling is impossible and irreversible, and the fastest development of the baby brain is in the first three months after birth, and then develops slowly. Therefore, good sleeping is very important for the neural development of infants. Many foreign scholars believe that a good sleeping awakening cycle combination mode can promote the development of infant sleep cycle to maturity mode, which is of great significance for infant brain development [7]. Newborns need 16 to 20 hours of sleeping time every day. Premature infants leave the mother's body too early, so the development of various systems, especially the nervous system, is still not perfect. Thus, the most of time after birth are in sleeping state. And then, sleep time and sleep pattern change with age. Jiang Fan [8] and others, according to epidemiological surveys of sleep time of infant from 1 month 
to 5 years old in Shanghai, pointed that the average sleep time of 1-months-old infant per day was 14.4 hours, and the sleep time gradually decreased with the increase of infant age. With the increase of the age of the baby, the average daily sleep time decreased from $6.9 \mathrm{~h}$ in the neonatal period to $3.8 \mathrm{~h}$ of the 6months-old infant, while the average sleep time in the daytime at 12 months was only $2.8 \mathrm{~h}$, but the night sleeping time of each age group did not change significantly. Foreign reports showed that during the first year of growth and development of infants, infant sleep gradually becomes continuously and mainly sleeps at night [9]. As a recombination state of brain function, sleep can consolidate memory and ensure that the brain performs its best function and is closely related to the energy conservation of the body. Compared with adults, sleep has special significance for infants. It is the necessary foundation for infant growth and development, cognition, emotion, behavioral development, and related immune function maturity. The development of sleep on various systems of infants, including endocrine system and body temperature regulation, cardiac respiratory system and neural developmental system have profound effects. Good sleep can enlarge the size of the cerebral cortex, increase synaptic connections, and accelerate the formation of the innervation of the thalamus cortex and the inner cortex [10]. Some experimental studies abroad have found that sleep can promote the development of the nervous system. For example, a study on newborn rats found that mice growing in a good sleep environment have increased cerebral cortex and produced more synaptic connections, but the rats deprived of eye movement sleep (REM) did not have the mentioned phenomenon [11]. Quiet sleep is also important for the development of the nervous system. The maturation process of quiet sleep is synchronized with the cycle of the formation of the thalamus cortex, the innervation of the inner cortex, and the increase in synaptic connections [12]. During quiet sleep time, the neural activity in waking stage is reactivated, meaning that the information obtained during the awake period can be further enhanced when in the quiet sleep state.

\section{RELATIONSHIP BETWEEN INFANT TEMPERAMENT CHARACTERISTICS AND SLEEP TIME}

Temperament is the external manifestation of infants' behavioral development, and it is a psychological indicator to measure the differences in individual behaviors of infants and young children. One possibility for temperament-affecting behavior is its regulation [13]: on the one hand, it directly regulates stress from children, and on the other hand, it regulates external stress in children. Thomas and Chess argue that temperament is a regulatory variable in a similar biopsychosocial model determined by neurobiology, which regulates between external stimuli and brain responses expressed by emotions, thoughts, and individual behavior effect. Temperament is also related to the sleep of infants. $\mathrm{Li}$ Xia et al [14] conducted a study on the relationship between sleep behavior and temperament of infants aged 3 to 6.5 years old. The study showed the sleep behavior of infants is closely related to the type of temperament, and temperament type infants have different sleep behaviors. Studies have shown that there are differences in sleep time between infants with different temperament types, and the temperament dimension is also related to sleep time. Xie Liangliang et al [15] pointed out that the time required for difficult infants and slow-starting infants to fall asleep again after waking up is longer than easyto-maintain infants and intermediate infants, and intermediate infants and difficult infants have shorter sleep time during the daytime than slow start-up infants, intermediate temperament infants have shorter sleep time in all day than infants with slower start-up infants and easy-to-maintain infants. Also, difficult temperament infants have shorter sleep time in all day than infants who are easy-to-maintain infants. Studies abroad have found that in babies time, difficult temperament is associated with short duration of sleep of children, and difficult temperament is related to sleep problems, specific performance: with difficult temperament type, preschool children tend to be more sensitive, irritable and vulnerable to the changing external environment, with strong disturbance, crying and intense reaction, and such children are more likely to wake up at night [16]. Studies in China have shown that children with poor sleep quality have a higher frequency of difficult temperament than children with good sleep quality, and are more likely to lose temper and score higher in emotional and behavioral problems [17]. A representative study abroad also showed that difficult temperament is a significant and separate risk factor for poor sleep rhythm, and points out avoidance, adaptability, emotional essence, persistence and the time required for infants to wake up again presented a negative correlation [18]. Zhang Fenghua et al [19] found that the evasiveness and response threshold were negatively correlated with the total sleep time of infants at night, that is, infant temperament tends to be more sensitive to new stimuli, new environment, and sensitive to light, noise, temperature, etc, with shorter total sleep time at night. Studies abroad have also shown that children who are arousal, are less likely to respond than children who are sleepy [20]. Activity level and avoidance were negatively correlated with the total sleep time of premature infants at night and daytime, that is, premature infants tend to have high activity levels, new stimuli, new environment retreat, with less total sleep time per day.

\section{PROSPECT OF RELATIONSHIP BETWEEN INFANT TEMPERAMENT AND SLEEP}

At present, there are few studies on the correlation between infant temperament characteristics and sleep relationship at home and abroad. In China, there are many studies on sleep in school-age children, but there are relatively few sleep studies on infants. The research methods are mostly retrospective research using parental questionnaires. Foreign studies on infant sleep are mostly recorded by EEG method, which can objectively observe the sleep awakening cycle of infants. The activity recorder, as a non-invasive and continuous recording method, can be effectively used for continuous sleep monitoring in the family environment, and the evaluation results are consistent with the behavior observation and polysomnography monitoring results. Studying the relationship between different temperament dimensions, temperament type characteristics and sleep behavior of infants helps us to find reliable early intervention methods based on the results of temperament assessment to help them establish a day and night sleep cycle and improve infant sleep to promote infant development. 


\section{REFERENCES}

[1] Pan Linglin, Huang Linying, Yang Biyun, et al. Study on the effect of infant temperament on infant sleep[J]. Chinese Journal of Women and Children Health Research, 2009, 20(2):132-134.(In Chinese).

[2] Parker S J, Barrett D E. Maternal Type A behavior during pregnancy, neonatal crying, and early infant temperament: do type A women have type A babies?[J]. Pediatrics, 1992, 89(3):474-9.

[3] Crockenberg S C, Leerkes E M. Infant temperament moderates associations between childcare type and quantity and externalizing and internalizing behaviors at $21 / 2$ years.[J]. Infant Behavior \& Development, 2005, 28(1):20-35.

[4] Yang Shaoping, Wu Hanrong, Zhang Bin, et al. Study on the relationship between sleep disorders and temperament in preschool children in Wuhan[J]. Chinese School Health, 2006, 27(9):739-740. (In Chinese).

[5] Wang Baozhu, Pan Jianping, Zheng Ping. The influence of early education on the social life ability of children with different temperament types[J]. Chinese General Practice, 2007, 10(21):17951796. (In Chinese).

[6] Tao Citizen, Chen Jingdong, Fang Hongying, et al. Relationship between temperament and sleep disorders in school-age children[J]. Anhui Medical Journal, 2010, 31(6):668-670. (In Chinese).

[7] Philbrook L E, Teti D M. Bidirectional associations between bedtime parenting and infant sleep: Parenting quality, parenting practices, and their interaction.[J]. J Fam Psychol, 2016, 30(4):431-441.

[8] Jiang Fan, Jin Xingming, Jiang Yilian, et al. Discussion on treatment methods related to sleep initiation in infants and young children[J]. Chinese Journal of Practical Pediatrics, 2005, 20(9):534-536. (In Chinese).

[9] Figueiredo B, Dias C C, Pinto T M, et al. Infant sleep-wake behaviors at two weeks, three and six months[J]. Infant Behavior \& Development, 2016, 44:169-178.

[10] Zhao Yantao, Huang Xiaona, Wang Huishan. Advances in research on the relationship between sleep and cognitive development in infants and young children[J]. Chinese Journal of Child Health, 2012, 20(9):814-816 (In Chinese).

[11] Miyazaki K, Itoh N, Ohyama S, et al. Development and characterization of mouse model for stress induced sleep disorder[J]. Neuroscience Research, 2011, 71(4):e172-e173.

[12] Liu Yucheng, Wang Huishan. Infant sleep and growth [M]. China Traditional Chinese Medicine Press, 2011. (In Chinese).

[13] Chen Yuying, Chen Jingyu, Pei Ping, et al. Progress in research on sleep status of infants and young children at home and abroad[J]. Chinese Journal of Women and Children Health Research,2016, 27(7):892-893. (In Chinese).

[14] Li Xia, Wang Hong, Xu Rong. Study on the relationship between sleep behavior and temperament in children aged 3 to 6.5 years[J]. Chinese Journal of Behavioral Medicine and Brain Science, 2000, 9(3):215-215. (In Chinese).

[15] Xie Liangliang, Cao Hui, Gu Chunli, et al. Relationship between sleep problems and temperament in infants at 6 months of age[J]. Maternal and Child Health Care in China, 2015, 30(11):1694-1697. (In Chinese).

[16] Moser M H, Denham S A. Infant Temperament: Stability, Parental Concurrence, and Relations to Mother-Infant Attachment.[J]. Attachment Behavior, 1989:10.

[17] Fang Feng, Wang Yali, Chen Guojun, et al. Analysis of sleep quality and emotional status in infants and young children $[\mathrm{J}]$. Chinese Journal of Child Health,2008, 16(4):471-472. (In Chinese).

[18] Judge M P, Chang L, Lammi-Keefe C J. Evidence of developmental continuity from birth to 1 year: sleep, temperament, problem solving, and recognition memory[J]. Adv Neonatal Care, 2015, 15(2):125-133.

[19] Zhang Fenghua, Liao Canhui, Wang Huixin, et al. Study on the relationship between early sleep/wakefulness and temperament in infants[J]. Chinese Journal of Clinicians (Electronic Edition),2014(12):99-102. (In Chinese).

[20] Jian N, Teti D M. Emotional availability at bedtime, infant temperament, and infant sleep development from one to six months.[J]. Sleep Medicine, 2016, 23:49-58. 